\title{
Editorial: Dietary Strategies for Healthy Aging-Caloric Restriction and Beyond
}

\author{
Sebastian J. Hofer ${ }^{1}$ and Sergio Davinelli ${ }^{2 *}$ \\ ${ }^{1}$ Institute of Molecular Biosciences, NAWI Graz, University of Graz, Graz, Austria, ${ }^{2}$ Department of Medicine and Health \\ Sciences "V. Tiberio", University of Molise, Campobasso, Italy
}

Keywords: caloric restriction, aging, nutrition, healthspan, caloric restriction mimetic

\section{Editorial on the Research Topic}

\section{Dietary Strategies for Healthy Aging-Caloric Restriction and Beyond}

Ample evidence generated over the past two decades underlines the crucial role of nutrition to achieve healthy aging. Numerous dietary strategies and food components have increasingly been investigated as interventions to preserve function and compress morbidity and disability to a period later in life. Findings from preclinical investigations, as well as cross-sectional, longitudinal, and intervention studies indicated that well-established 'holistic' healthy diets [e.g., DASH (1) and Mediterranean diets $(2,3)]$ and specific dietary components [e.g., polyamines (4), and polyphenols (5)] may exert health-promoting effects during aging.

Among these dietary strategies, caloric restriction (CR), an ambiguous term often synonymously used with energy restriction (ER), has proven to be a very robust and broadly applicable intervention that affects aging-relevant molecular pathways and induces a range of beneficial effects involved in the maintenance of physiological functions throughout the life course $(6,7)$. Generally, CR is defined as a reduction in daily caloric intake without malnutrition. Different dietary alternative strategies have been developed to produce CR-like effects. Prominent examples include intermittent fasting (IF) and alternate-day fasting (ADF) (8). However, since the real-life applicability of CR is hardly sustainable in the long-term, the development of pharmacological mimetics of $\mathrm{CR}$ has gained considerable attention $(9,10)$. Multiple screenings have been performed or are currently taking place to identify synthetic or natural molecules that can be used to mimic CR phenotypes.

This Research Topic contains 11 articles covering the specific parts of the abovementioned aspects.

Frailty is a prevalent age-related clinical condition generally recognized as a state of high vulnerability to adverse health outcomes (11). Although its etiology remains poorly understood, specific dietary regimens may prevent, delay, or even reverse this condition. Liu et al. summarize evidences on the impact of CR and alternative approaches on frailty syndrome, highlighting potential underlying mechanisms. Besides frailty, older adults are particularly vulnerable to cardiometabolic disease and unhealthy diet is a leading risk factor for the development of cardiometabolic disease (12). Perry et al. have examined the response of a caloric-restricted DASH (Dietary Approaches to Stop Hypertension) diet on parameters of cardiometabolic health in a cohort of sedentary obese older adults. They found that a DASH-like diet with restricted calories is an effective approach to improve cardiovascular, metabolic, and inflammatory biomarkers. Besides calories, macronutrients play an important role in shaping a diet's effects on health and aging (13). Dietary fiber is considered to contribute no calories to our diet (14) and its intake is associated with lower risk of type 2 diabetes, cardiovascular disease, and weight gain. However, the antihypertensive

Received: 31 January 2022 Accepted: 08 February 2022 Published: 01 March 2022 
effect of dietary fiber intake has not been elucidated until now. Results from the SWAN study published in this e-collection revealed that intake of dietary fiber from grains contributes to a lower risk of hypertension in midlife women.

In this scenario, Voglhuber et al. provide an excellent overview of alternatives to $\mathrm{CR}$ (e.g., IF, $\mathrm{ADF}$, and the Mediterranean diet) that could mimic the cardiometabolic benefits of $\mathrm{CR}$ in animal models and humans. The authors also summarize emerging potential $\mathrm{CR}$ mimetics, such as spermidine, $\mathrm{NAD}^{+}$precursors, and metformin, highlighting the mechanism underpinning their cardiometabolic and healthpromoting effects. Similarly, Stadler and Marsche provide an insightful overview, detailing how dietary strategies and various nutritional components may improve the atheroprotective functions of high-density lipoproteins (HDL). They discuss the clinical efficacy of Mediterranean diet, CR, and IF to increase HDL functionality and promote cardiovascular health. Furthermore, these authors address this topic by focusing on phenolic compounds that could exert positive effects on HDL function.

As briefly discussed in the articles by Voglhuber et al., as well as Stadler and Marsche, CR and fasting applications in real-life contexts harbor significant challenges. Hence, the concept of CR mimetics (CRMs) was developed to identify bioactive substances that mimic the molecular effects induced by CR (9, 10, 15). Recently, this research field has gained an increasing scientific interest. Therefore, in Hofer et al. we have performed a comprehensive literature review describing a wide range of naturally occurring substances with CRmimicking properties, along with their dietary sources, intake levels and the current state of clinical research. Despite challenges in assimilating experimental findings into clinical treatments, CRM candidates, such as polyamines, polyphenols, $\mathrm{NAD}^{+}$precursors, and glycolytic inhibitors, appear to prolong life- and healthspan in model organisms. Accordingly, the consumption of CRMs may elicit numerous disease-protecting activities, including neuroprotective effects. In line, the paper from de Vries et al. presents a systematic review and meta-analysis on the brain-enhancing effects of polyphenols, a promising source of potential CRMs. They included 66 randomized clinical trials with participants aged 40 years or older. The results indicate that short- and moderateterm supplementation with some classes of polyphenols may improve working and episodic memory in non-pathological and pathological aging.

\section{REFERENCES}

1. Chiavaroli L, Viguiliouk E, Nishi SK, Blanco Mejia S, Rahelić $\mathrm{D}$, Kahleová $\mathrm{H}$, et al. DASH dietary pattern and cardiometabolic outcomes: an umbrella review of systematic reviews and meta-analyses. Nutrients. (2019) 11:338. doi: 10.3390/nu1102 0338

2. Davinelli S, Trichopoulou A, Corbi G, De Vivo I, Scapagnini G. The potential nutrigeroprotective role of Mediterranean diet and its functional components on telomere length dynamics. Ageing Res Rev. (2019) 49:110. doi: 10.1016/j.arr.2018.11.001
Although religious fasting is practiced by millions of believers around the world, scarce data exist on its impact on human health. For centuries, humans have adopted various forms of religious fasting as regimens of spiritual purification. Baháí fasting is a specific form of intermittent dry fasting characterized by abstaining from food and liquids during daylight hours every year in March for 19 consecutive days. Koppold-Liebscher et al. conducted a prospective exploratory cohort study in Baháí volunteers to evaluate safety and effects of Baháí fasting on hydration, metabolism, and circadian clock. The study revealed that this form of fasting is safe with no negative effects on hydration biomarkers. Furthermore, Bahái fasting appears to improve fat metabolism and cause only transient alterations of circadian rhythms. Despite the relatively small number of studies on this topic, fasting treatments are also associated with detoxifying properties. Grundler et al. explored the effects of long-term fasting (10 days) on the excretion of heavy metals and glyphosate in 109 healthy subjects. The results of the study provide first insights into the changes in heavy metal excretion after fasting and show a reduction in the urinary levels of arsenic and nickel, as well as a reduction in hair lead levels.

The opinion article from Ostojic discusses the importance of the creatine transporter (CT1), which is located on the plasma membrane of various energy-demanding cells and whose dysfunction is associated with movement and behavior disorders. A potential but barely explored cellular effect of CR could be an increase in CT1 activity, facilitating creatinine uptake.

Finally, Wahl and LaRocca present a detailed review of the transcriptomic effects of healthy nutritional interventions, including $\mathrm{CR}$, IF, ADF, prolonged fasting, time-restricted feeding, and protein and amino acid restriction. They describe gaps in the research, highlighting the importance of transcriptome/multi-omic studies to better understand the effects of these dietary interventions.

Despite the richness and importance of the topics covered in the papers published in this e-collection, the complex impact of $\mathrm{CR}$ and CR-alternatives on aging and health is still insufficiently understood and there are many aspects to be clarified in this fascinating research field.

\section{AUTHOR CONTRIBUTIONS}

Both authors listed have made a substantial, direct, and intellectual contribution to the work and approved it for publication. Clin Nutr. (1995) 61:1402S-6S. doi: 10.1093/ajen/61.6.1402S

4. Madeo F, Hofer SJ, Pendl T, Bauer MA, Eisenberg T, Carmona-Gutierrez D, et al. Nutritional aspects of spermidine. Ann Rev Nutr. (2020) 40:13559. doi: 10.1146/annurev-nutr-120419-015419

5. Yessenkyzy A, Saliev T, Zhanaliyeva M, Masoud AR, Umbayev B, Sergazy S, et al. Polyphenols as caloric-restriction mimetics and autophagy inducers in aging. Res Nutr. (2020) 12:1344. doi: 10.3390/nu12051344

6. Di Francesco A, Di Germanio C, Bernier M, de Cabo R. A time to fast. Science. (2018) 362:770-5. doi: 10.1126/science.aau2095 
7. Flanagan EW, Most J, Mey JT, Redman LM. Calorie restriction and aging in humans. Аnnu Rev Nutr. (2020) 40:10533. doi: 10.1146/annurev-nutr-122319-034601

8. Hofer SJ, Carmona-Gutierrez D, Mueller MI, Madeo F. The ups and downs of caloric restriction and fasting: from molecular effects to clinical application. EMBO Mol Med. (2021) 14:e14418. doi: 10.15252/emmm.202114418

9. Madeo F, Carmona-Gutierrez D, Hofer SJ, Kroemer G. Caloric restriction mimetics against age-associated disease: targets, mechanisms, and therapeutic potential. Cell Metab. (2019) 29:592-610. doi: 10.1016/j.cmet.2019.01.018

10. Ingram DK, Zhu M, Mamczarz J, Zou S, Lane MA, Roth GS, et al. Calorie restriction mimetics: an emerging research field. Aging Cell. (2006) 5:97108. doi: 10.1111/j.1474-9726.2006.00202.x

11. Davinelli S, Corbi G, Scapagnini G. Frailty syndrome: a target for functional nutrients? Mech Ageing Dev. (2021) 195:111441. doi: 10.1016/j.mad.2021.111441

12. Lloyd-Jones DM, Hong Y, Labarthe D, Mozaffarian D, Appel LJ, Van Horn L, et al. Defining and setting national goals for cardiovascular health promotion and disease reduction: the American Heart Association's strategic Impact Goal through 2020 and beyond. Circulation. (2010) 121:586613. doi: 10.1161/CIRCULATIONAHA.109.192703

13. Solon-Biet SM, Mitchell SJ, de Cabo R, Raubenheimer D, Le Couteur DG, Simpson SJ. Macronutrients and caloric intake in health and longevity. $J$ Endocrinol. (2015) 226:R17-28. doi: 10.1530/JOE-15-0173
14. Turner ND, Lupton JR. Dietary Fiber. Adv Nutr. (2011) 2:151-2. doi: 10.3945/an.110.000281

15. Davinelli S, De Stefani D, De Vivo I, Scapagnini G. Polyphenols as caloric restriction mimetics regulating mitochondrial biogenesis and mitophagy. Trends Endocrinol Metab. (2020) 31:536-50. doi: 10.1016/j.tem.2020.02.011

Conflict of Interest: The authors declare that the research was conducted in the absence of any commercial or financial relationships that could be construed as a potential conflict of interest.

Publisher's Note: All claims expressed in this article are solely those of the authors and do not necessarily represent those of their affiliated organizations, or those of the publisher, the editors and the reviewers. Any product that may be evaluated in this article, or claim that may be made by its manufacturer, is not guaranteed or endorsed by the publisher.

Copyright $\odot 2022$ Hofer and Davinelli. This is an open-access article distributed under the terms of the Creative Commons Attribution License (CC BY). The use, distribution or reproduction in other forums is permitted, provided the original author(s) and the copyright owner(s) are credited and that the original publication in this journal is cited, in accordance with accepted academic practice. No use, distribution or reproduction is permitted which does not comply with these terms. 